\title{
Evolution of an energy dissipating protein domain indicates shared origin for ancient metabolisms and a self- organizing mechanism
}

\author{
BENNETT JAMES KAPILI AND ANNE E DEKAS
}

Stanford University

Presenting Author: kapili@stanford.edu

Organisms couple the dissipation of environmental free energy to processes that support further energy dissipation. Here, we study the evolutionary history of an ancient ATP hydrolase to understand how the emergence of an energy dissipating protein domain supported the emergence of complex metabolisms. We combine machine learning, phylogenetic, and comparative structure approaches - relying on hundreds of researchers' previous works for biochemical context - to determine that the emergence and evolution of a P-loop ATPase resulted in mechanisms for: (1) releasing stored energy to provide a chemical reaction flux and (2) generating spatial information through dynamics consistent with Turing's reaction-diffusion model. We find that a shared arrangement of homologous conserved sequence elements underpins the proteins affecting both mechanisms, which were applied to: (1) the biosynthesis of different cofactors and pigments, (2) ensuring cell division occurs at the midline, and (3) the partitioning of chromosomes and plasmids to opposite cell poles prior to division. In particular, our phylogenetic estimates suggest that the proteins that help assemble the FeMo-co and P-cluster of nitrogenase, cofactor F430, [4Fe4S] clusters, the active site of [NiFe]hydrogenase, and affect the greening of chlorophyll are evolutionary descendants of the proteins that assemble the inorganic A- and C-clusters of the Wood-Ljungdahl pathway. Collectively, our results indicate that the emergence and evolution of a specific energy dissipating protein domain directly supported the emergence of numerous geobiologically-important metabolisms, as well as mechanisms that increased the fidelity of cell division and genetic inheritance. 